\title{
Rendimiento del trigo cv. 'centenario' bajo diferentes densidades de siembra en un sistema de riego presurizado
}

\author{
Wheat yield cv. 'centenario' under different planting densities in a pressurized irrigation system
}

Rendimento de trigo 'centenário' em diferentes densidades de semeadura em um sistema de irrigação pressurizado

\section{Luis Felipe Solis Rosas Diaz}

lfsolisrosas@gmail.com

https://orcid.org/0000-0002-7244-8272

Universidad Nacional San Luis Gonzaga de Ica, Ica, Perú

\author{
Jorge Tejada Soraluz \\ jorgetejada@lamolina.edu.pe \\ https://orcid.org/0000-0002-2102-1578
}

Universidad Nacional Agraria La Molina. Lima, Perú

\author{
Jorge Jiménez Dávalo \\ jjimenezd@lamolina.edu.pe \\ https://orcid.org/0000-0002-2366-6310 \\ Universidad Nacional Agraria La Molina. Lima, Perú
}

Artículo recibido 02 de abril 2021 / Arbitrado y aceptado 16 de abril 2021 / Publicado 04 de mayo 2021

\section{RESUMEN}

El experimento se llevó a cabo en la provincia y departamento de Ica, Perú (costa central) bajo un sistema de riego presurizado, en el cultivo de trigo (Triticum aestivum ssp. aestivum) cv. 'centenario', en la cual hay mucha área bajo riego con agua de alta salinidad, el trigo por su resistencia a la alta salinidad del agua es una alternativa viable. Se utilizó el Diseño de Bloques Completamente al Azar con arreglo factorial, con 9 tratamientos y 4 repeticiones. Aplicando 2 factores, distanciamiento entre semillas (S) S0.5 $\mathrm{cm}, \mathrm{S} 1.0 \mathrm{~cm}$ y $\mathrm{S} 2.0 \mathrm{~cm}$ y número de líneas (L) L2, L4 y L6. Para la comparación de medias se utilizó la prueba Duncan al 0.05 de significancia. En todas las variables en el análisis de varianza no se obtuvo significancia en la interacción entre los factores S y L. En rendimiento por ha-1 con el distanciamiento entre semillas a 1.0 $\mathrm{cm}$ y $2.0 \mathrm{~cm}$ (S1 y S2) se obtuvo 11513.89 y $11044.44 \mathrm{~kg}$ por ha-1 respectivamente y con cuatro líneas de trigo (L4) se obtuvo 12343.75.

El mayor número de espigas por $\mathrm{m}-2$ se obtuvo al sembrar entre semillas con 0.5 $\mathrm{cm}$ y $1.0 \mathrm{~cm}$ con 681.42 y 653.33 espigas por $\mathrm{m}-2$. Con 4 y 6 líneas se obtuvo 727.67 y 693.50 espigas por m-2. Para obtener los mejores rendimientos y economizar semillas se debe emplear los factores S2 y L4. El mayor número de espigas por $\mathrm{m}-2$ se obtuvo con los factores S0.5, S1.0, L4 y L6.

Palabras clave: Costa Central, Densidad de siembra, riego por goteo, trigo, Triticum aestivum ssp. Aestivum
ABSTRACT

The experiment was carried out in the province and department of Ica, Perú (central coast) under a pressurized irrigation system, in the cultivation of wheat (Triticum aestivum ssp. Aestivum) cv. 'Centennial', in which there is a lot area under irrigation with high salinity water, wheat due to its resistance to high water salinity is a viable alternative. The Completely Random Block Design was used with factorial arrangement, with 9 treatments and 4 repetitions. Applying 2 factors, spacing between seeds (S) S0.5 cm, S1.0 cm and S2.0 $\mathrm{cm}$ and number of lines (L) L2, L4 and L6. For the comparison of means the Duncan test was used at 0.05 of significance. In all the variables in the analysis of variance, there was no significance obtained in the interaction between the factors $S$ and L.In yield per ha-1 with the spacing between seeds at $1.0 \mathrm{~cm}$ and $2.0 \mathrm{~cm}$ (S1 and S2), 11513.89 and $11044.44 \mathrm{~kg}$ per ha-1 were obtained respectively and with four lines of wheat (L4) 12343.75 were obtained. The highest number of spikes per $\mathrm{m}-2$ was obtained when sowing between seeds with $0.5 \mathrm{~cm}$ and $1.0 \mathrm{~cm}$ with 681.42 and 653.33 spikes per m-2. With 4 and 6 lines, 727.67 and 693.50 spikes per $\mathrm{m}-2$ were obtained. To obtain the best yields and save seeds, factors S2 and L4 should be used. The highest number of spikes per m-2 was obtained with the factors S0.5, S1.0, L4 and L6.

Key words: Central Coast, Seed density, drip irrigation, wheat, Triticum aestivum ssp. Aestivum

\section{RESUMO}

O experimento foi conduzido na província e departamento de Ica, Peru (costa central) sob um sistema de irrigação pressurizado, no cultivo de trigo (Triticum aestivum ssp. Aestivum) cv. 'Centenário', em que existe uma grande área sob irrigação com água de alta salinidade, o trigo devido à sua resistência à alta salinidade da água é uma alternativa viável. Foi utilizado o Delineamento em Bloco Completamente Aleatório com arranjo fatorial, com 9 tratamentos e 4 repetições. Aplicando 2 fatores, espaçamento entre sementes (S) $S 0.5 \mathrm{~cm}, 51.0 \mathrm{~cm}$ e $S 2.0 \mathrm{~cm}$ e número de linhas (L) L2, L4 e L6. Para a comparação das médias, foi utilizado o teste de Duncan a 0.05 de significância. Em todas as variáveis da análise de variância, não foi obtida significância na interação entre os fatores $\mathrm{S}$ e L.Na produtividade por ha- 1 com 0 espaçamento entre as sementes de $1.0 \mathrm{~cm}$ e $2.0 \mathrm{~cm}$ (S1 e S2), foram obtidos 11513.89 e $11044.44 \mathrm{~kg}$ por ha-1 respectivamente e com quatro linhagens de trigo (L4) 12343.75. O maior número de espigas por $\mathrm{m}-2$ foi obtido na semeadura entre sementes com $0.5 \mathrm{~cm}$ e $1.0 \mathrm{~cm}$ com 681.42 e 653.33 espigas por $\mathrm{m}-2$. Com 4 e 6 linhas, foram obtidos 727.67 e 693.50 picos por m-2. Para obter os melhores rendimentos e economizar sementes, os fatores S2 e L4 devem ser usados. O maior número de pontas por $\mathrm{m}-2$ foi obtido com os fatores S0.5, S1.0, L4 e L6.

Palavras-chave: Costa Central, Densidade de semeadura, irrigação por gotejamento, trigo, Triticum aestivum ssp. Aestivum 


\section{INTRODUCCIÓN}

Rendimiento del trigo a nivel mundial está basada en su mayoría en riego bajo secano, lo cual no es una realidad para localidad peruana, debido a que desconoce teniendo como premisa que el rendimiento del cultivo de trigo está influenciado directamente a su densidad como reportaron Suaste (1) que obtuvieron el máximo rendimiento de trigo con una densidad

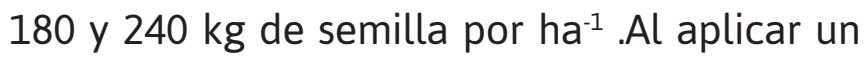
sistema de riego presurizado (tecnología alta), también, se debe fertilizar y regar óptimamente para obtener rendimientos altos y justifique la inversión como lo reportan Lernen (2) que la que fertilidad nitrogenada y densidades siembra actuaron independientemente sobre producción de materia seca, aunque el aporte de nitrógeno aumenta la materia seca. Aparte también el emplear altas densidades ayuda a la mitigación de maleza como lo reportaron Weiner (3) quienes con 400 semillas por $\mathrm{m}^{-2}$ redujeron la biomasa de las malezas aumentando el rendimiento hasta en un $60 \%$ más.

La problemática principal en Ica, de una localidad en Perú, Villacuri es que la expansión agrícola del lugar mencionado está limitada por la falta de agua con calidad suficiente para el riego. En la actualidad se encuentran grandes extensiones agrícolas abandonadas por falta de agua de buena calidad y continúa aumentando las áreas que debido a la falta del recurso hídrico de calidad y el trigo es una alternativa por su resistencia a la salinidad.

Además, el uso de semilla aptas para distribuir por el departamento de cereales de la Universidad Nacional Agraria la Molina cv "Centenario", influenciaron en un óptimo desarrollo del cultivo como lo reportan Abati (4).
Uno de los factores más importantes es obtener un alto número de espigas por $\mathrm{m}^{-2}$ por que la esta investigación también determino y se puede comprar con lo reportado por Lerner (2) que usando la densidad de 600 plantas por $\mathrm{m}^{-2}$ el número de espigas fue mayor.

El principal objetivo de este estudio es evaluar el rendimiento del trigo cv "Centenario" en un sistema de riego presurizado y diferentes densidades de siembra en condiciones de la costa Central del Perú mediante el estudio de la respuesta en rendimiento del trigo cV "Centenario" a diferentes distanciamientos entre semillas y número de líneas.

Al investigar en estas áreas con agua de mala calidad y obtener rendimientos altos a bajo costo nos permite ser competitivos económicamente y que el Perú consuma trigo nacional con el fin de remplazar en un futuro a la importación de esta materia prima.

El trigo, si bien es cierto, es un commodity sus precios internacionales son estables pero bajos, por lo que se tiene que obtener altos rendimientos por hectárea para que sustente los gastos de aplicar alta tecnología obligada por tener suelos arenosos que implican el uso de riego por goteo compitiendo con trigo bajo sistema de secano que es en la mayoría del mundo.

\section{MATERIALES Y MÉTODOS}

La semilla del trigo (Triticum aestivum ssp. aestivum)cv 'Centenario' se obtuvo del Programa de Investigación y Proyección Social en Cereales y Granos Nativos de la UNALM (Universidad Nacional Agraria La Molina). El experimento se llevó a cabo en el lote experimental del Fundo "San Felipe" que se encuentra localizada en el km 271 Panamericana sur, distrito Salas, provincia y departamento de Ica, Perú, en el 
período comprendido entre 31 setiembre del año 2019 y 22 de enero de 2020.

El método de muestreo fue cosechando con segadora manual luego se pasó por una maquina trilladora estacionaria obteniendo las observaciones dependiendo la etapa del proceso. Se llevó a cabo mediante una investigación de campo, en donde se recopilaron antecedentes, y procesar los datos estadísticos, además se realizó una revisión documental de artículos sobre las variables estudiadas.

El procedimiento que se llevó a cabo basada en las variables que a bien se determinaron fue: 1 ). Rendimiento ( $\mathrm{kg} \mathrm{ha}^{-1}$ ): La cual se obtuvo luego de trillado del trigo seco y pesado en balanza analítica por muestra; 2). Numero espigas por $\mathrm{m}^{-2}$ : Se contó directamente en la zona experimental del cultivo; 3). Peso una espiga solo grano (g): Que resulta de dividir el peso solo grano por $\mathrm{m}^{-2}$ entre número de espigas por $\mathrm{m}^{-2}$ esta información se recopilo del campo directamente; 4). Peso de espigas con grano por $\mathrm{m}^{-2}(\mathrm{~g})$ : Antes de trillar se pesó cada muestra (espigas con su grano) con una balanza analítica y se llevó el resultado a $\left.\mathrm{m}^{-2} ; 4\right)$. Peso de una espiga con grano (g): Se dividió el peso de espigas con grano en un $\mathrm{m}^{-2}$ entre su número de espigas por $\mathrm{m}^{-2} ; 6$ ). Porcentaje peso del grano en relación a su espiga: Se dividió el peso promedio solamente del grano de una espiga sobre el promedio del peso de espiga más grano; 7 ). Peso de una espiga sin grano (g): Luego de pesar la muestra trillada en la balanza analítica se promedió, se dividió entre el número de espigas y se obtuvo el peso de grano por espiga; 8). Altura de planta $(\mathrm{m})$ : Con Wincha métrica se tomó varias alturas de plantas desde la base del suelo hasta la parte superior de la espiga, por cada muestra y luego se promedió.

El experimento fue instalado utilizando un sistema de riego por goteo, con distanciamiento entre cintas de 0.60 metros. Se puede apreciar que se puede llegar a cosecha más precoz que en lugares con menos temperatura (Tabla 1).

Tabla 1. Etapas fenológicas y operaciones en el cultivo de trigo.

\begin{tabular}{lccc}
\hline \multicolumn{1}{c}{ Descripción operación } & Fecha & $\begin{array}{c}\text { Días cumulados } \\
\text { después de siembra }\end{array}$ & m3 \\
\hline Riego de machaco & $25 / 09 / 2019$ & 0 & 480 \\
Siembra & $30 / 09 / 2019$ & 0 & 80 \\
Germinación & $7 / 10 / 2019$ & 7 & 280 \\
Desarrollo de las hojas & $22 / 10 / 2019$ & 22 & 750 \\
Macollaje & $6 / 11 / 2019$ & 37 & 900 \\
Encañado & $16 / 11 / 2019$ & 47 & 600 \\
Hinchamiento de las panículas o espigas & $1 / 12 / 2019$ & 62 & 900 \\
Espigado & $16 / 12 / 2019$ & 77 & 900 \\
Formación del fruto & $31 / 12 / 2019$ & 92 & 900 \\
Maduración de frutos y semillas & $10 / 01 / 2020$ & 102 & 400 \\
Cosecha & $20 / 01 / 2020$ & 112 & 200 \\
Total m3 & & & 5910 \\
\hline
\end{tabular}


El análisis de agua se realizó en el laboratorio Agriquem, cuyos resultados son: Conductividad eléctrica $3.56 \mathrm{mS} / \mathrm{cm}, \mathrm{pH}$ 7.92, alcalinidad 0.93 $\mathrm{meq} / \mathrm{l}$, nitratos $2.9 \mathrm{meq} / \mathrm{l}$, potasio $0.31 \mathrm{meq} / \mathrm{l}$, calcio $22.53 \mathrm{meq} / \mathrm{l}$, magnesio $4.32 \mathrm{meq} / \mathrm{l}$, sodio $9.31 \mathrm{meq} / \mathrm{l}$, boro $0.19 \mathrm{mg} / \mathrm{l}$, hierro $<0.05$ $\mathrm{mg} / \mathrm{l}$, manganeso $<0.05 \mathrm{mg} / \mathrm{l}$, zinc $<0.05 \mathrm{mg} / \mathrm{l}$, cloruros $21.82 \mathrm{meq} / \mathrm{l}$, sulfatos $8.51 \mathrm{meq} / \mathrm{l}$.

El análisis de suelo se realizó en el laboratorio "Valle Grande" instituto rural ubicado en la ciudad de Cañete cuyos resultados que corresponden a un suelo arenoso con Conductividad eléctrica (E.S) $7.97 \mathrm{dS} / \mathrm{m}, \mathrm{pH}$ : 7.92 , materia orgánica $<0.03$, nitrógeno total $<$ $0.0017 \%$; fósforo disponible 4.58 ppm, potasio disponible 115.54 ppm, carbonato de calcio $1.15 \%$. Cationes cambiables: Calcio 1.54 meq/100 gr, Magnesio 0.59 meq/100 gr, sodio $0.59 \mathrm{meq} / 100 \mathrm{gr}$, potasio $0.28 \mathrm{meq} / 100 \mathrm{gr}$ y P.S.I 19.67 \%, C.I.C.E 3.00 meq/100 gr. Sales solubles: Cloruro 50.42, sulfato $30.86 \mathrm{meq} / \mathrm{l}$, nitrato 2.91 $\mathrm{meq} / \mathrm{l}$, bicarbonato $1.80 \mathrm{meq} / \mathrm{l}$, calcio 45.05 $\mathrm{meq} / \mathrm{l}$, magnesio $8.76 \mathrm{meq} / \mathrm{l}$, sodio $31.43 \mathrm{meq} / \mathrm{l}$, potasio $0.49 \mathrm{meq} / \mathrm{l}$, boro $2.20 \mathrm{ppm}$.

Se utilizó la dosis de fertilización en relación a una hectárea fue de Nitrógeno (N) 263.24 kg, Fosforo (P2O5) 234.00 kg, Potasio (K2O) 168.61, Calcio (CaO) 65.00 kg, Magnesio (MgO) 52.00 kg,
Fierro (Fe) 6.50 kg, zinc (Zn) 6.50 kg, manganeso (Mn) $5.20 \mathrm{~kg}$ y boro (B) $6.50 \mathrm{~kg}$. Respecto a la sanidad del cultivo solo se realizó 2 aplicaciones: La primera aplicación con el producto splinter con materia activa spinosad $50 \mathrm{ml}$ por 200 lt de agua, también se aplicó Amistar con materia activa azoxistrobin a relación de $250 \mathrm{gr}$ por hectárea, estos se aplicaron con maxi cover con materia activa Polyether-Polymethylsiloxano a relación de a 100 cc por cilindro y BB5 con materia activa Ácido Ortofosfórico a relación de $50 \mathrm{ml}$ por 200 litros. Se aplicó en la etapa de inicio de macollaje. Para control de lepidópteros y Roya; La segunda aplicación se aplicó lo mismo que en la primera, pero ser le agrego Enzipron constituido de aminoácidos. Se aplicó en la etapa de espigado en la formación de la flor. Para el control de lepidópteros, roya, formar una buena flor y cuaja.

Se utilizó el Diseño de Bloques Completamente al Azar con arreglo factorial, con 9 tratamientos con 4 repeticiones. Utilizando 2 factores que fueron distanciamiento entre semillas S0.5, $S 1.0$ y $S 2.0 \mathrm{~cm}$ respectivamente y número de líneas L2, L4 y L6. Para la comparación de medias se utilizó la prueba Duncan con 0.05 de significancia. Cada tratamiento obedece a diferentes densidades (Tabla 2).

Tabla 2. Identificación de los tratamientos donde se muestra la densidad en $\mathrm{m}^{-2}$, numero de semillas y kilogramos de semilla asumiendo una hectárea.

\begin{tabular}{cccccc}
\hline Tratamientos & $\begin{array}{c}\text { Distanciamiento } \\
\text { entre semillas } \\
\mathbf{c m}^{\star}\end{array}$ & $\begin{array}{c}\text { Número de } \\
\text { líneas }^{\star *}\end{array}$ & $\begin{array}{c}\text { Numero de } \\
\text { semillas por } \mathbf{m - 2}\end{array}$ & $\begin{array}{c}\text { Numero de } \\
\text { semillas por } \\
\text { hectárea }\end{array}$ & $\begin{array}{c}\text { kilogramos de } \\
\text { semilla por } \\
\text { hectárea }\end{array}$ \\
\hline S 0.5 - L 2 & & 2.0 & 666.7 & 6666.7 & 307.0 \\
S 0.5 - L 4 & 0.5 & 4.0 & 1333.3 & 13333.3 & 613.0 \\
S 0.5 - L 6 & & 6.0 & 2000.0 & 20000000.0 & 920.0 \\
\hline
\end{tabular}




\begin{tabular}{|c|c|c|c|c|c|}
\hline Tratamientos & $\begin{array}{c}\text { Distanciamiento } \\
\text { entre semillas } \\
\text { cm }^{\star}\end{array}$ & $\begin{array}{l}\text { Número de } \\
\text { líneas }\end{array}$ & $\begin{array}{c}\text { Numero de } \\
\text { semillas por } m-2\end{array}$ & $\begin{array}{l}\text { Numero de } \\
\text { semillas por } \\
\text { hectárea }\end{array}$ & $\begin{array}{l}\text { kilogramos de } \\
\text { semilla por } \\
\text { hectárea }\end{array}$ \\
\hline S $1.0-$ L 2 & & 2.0 & 333.3 & 3333333.0 & 153.0 \\
\hline S $1.0-\mathrm{L} 4$ & 1,0 & 4.0 & 666.7 & 6666667.0 & 307.0 \\
\hline S $1.0-L 6$ & & 6.0 & 1000.0 & 10000000.0 & 460.0 \\
\hline$S 2.0-\mathrm{L} 2$ & & 2.0 & 166.7 & 1666667.0 & 77.0 \\
\hline $\mathrm{S} 2.0-\mathrm{L} 4$ & 2,0 & 4.0 & 333.3 & 3333333.0 & 153.0 \\
\hline S $2.0-\mathrm{L} 6$ & & 6.0 & 500.0 & 5000000.0 & 230.0 \\
\hline
\end{tabular}

*Distanciamiento entre semillas: 0.5 Siembra entre $0.5 \mathrm{~cm}, 1.0$ siembra entre $1 \mathrm{~cm}$ y 2.0 siembra entre $2 \mathrm{~cm}$

**Número de líneas: 2 una línea en ambos lados de la cinta de riego, 4 dos líneas en ambos lados de la cinta de riego y 6 tres líneas en ambos lados de la cinta de riego.

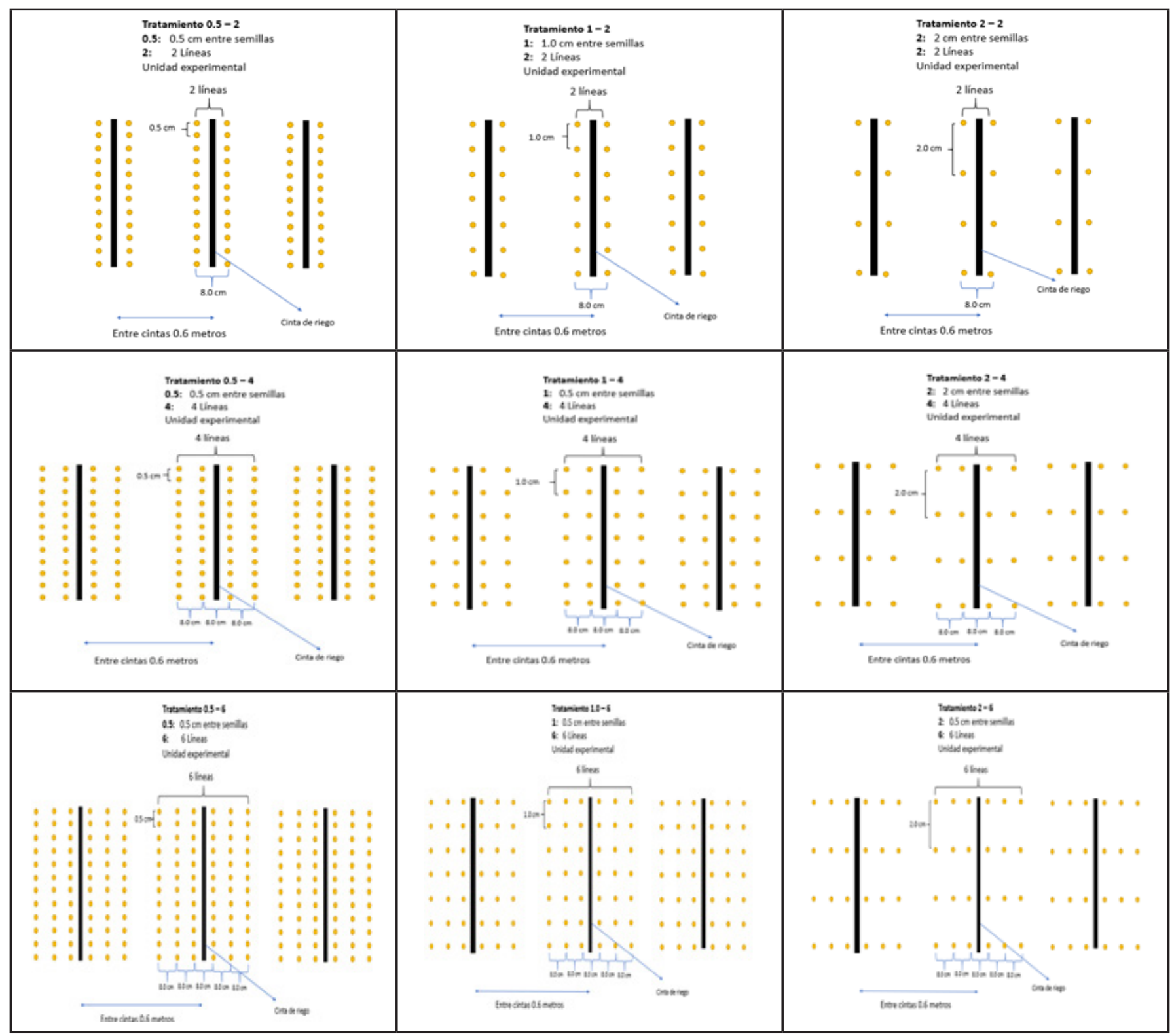

Figura 1. Esquema de siembra por tratamiento. 


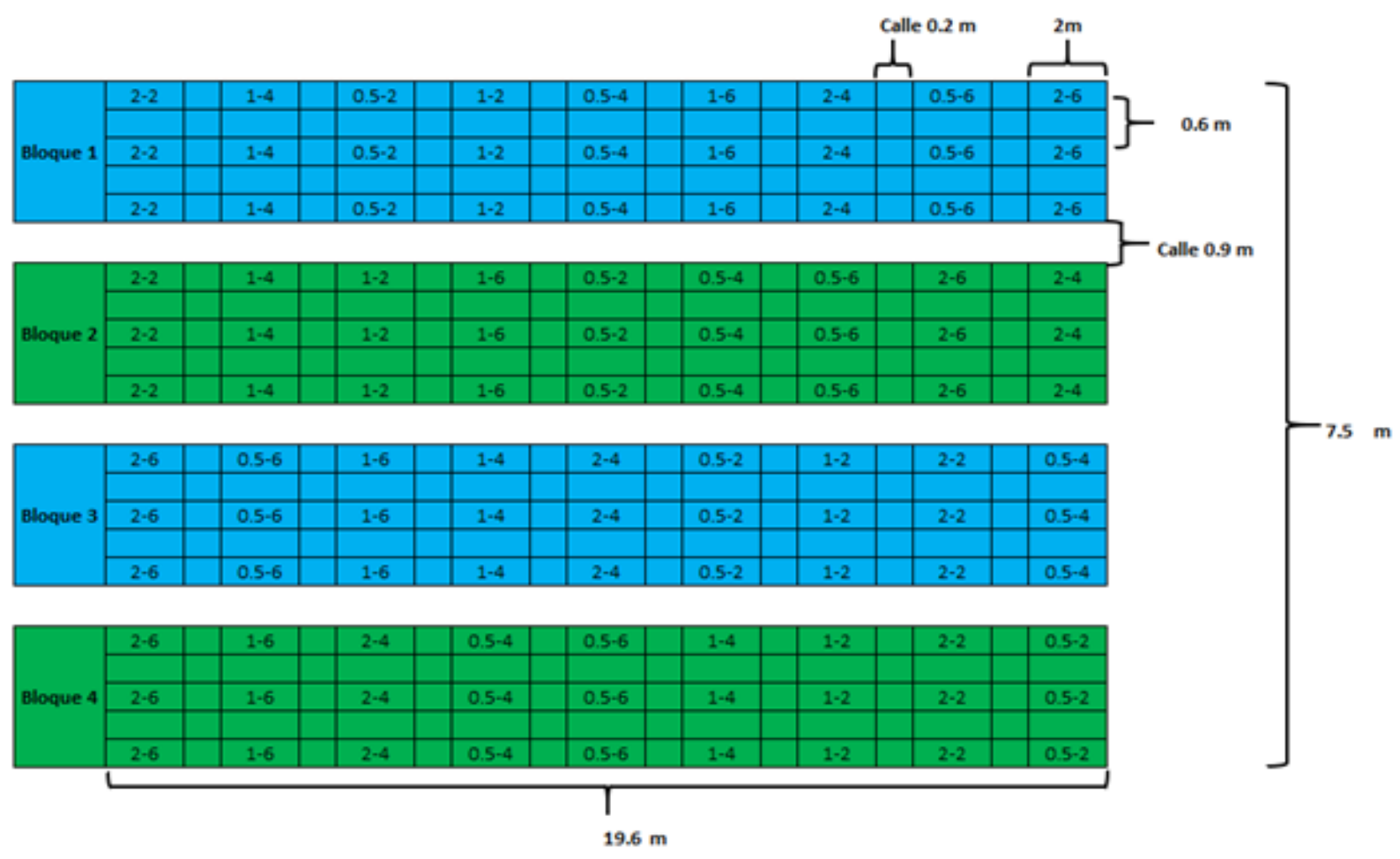

Figura 2. Croquis experimental.

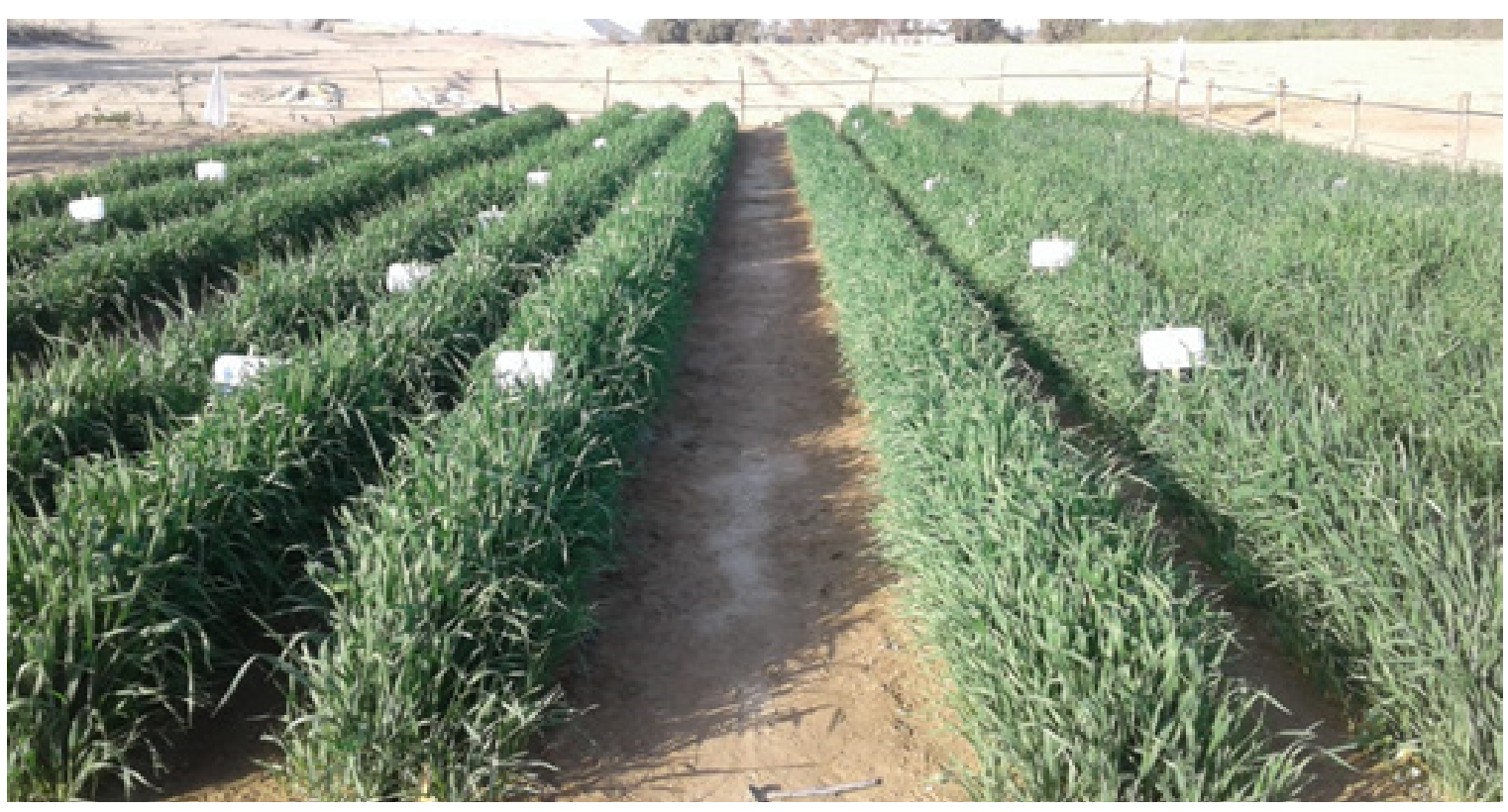

Figura 3. Macollaje, pre encañado del cultivo de trigo.

Se puede apreciar un buen macollaje y color de hoja a pesar de la alta conductividad del agua (Figura 3). Respecto a la maduración del cultivo se observa que viene de forma similar para todas las plantas (Figura 4). 


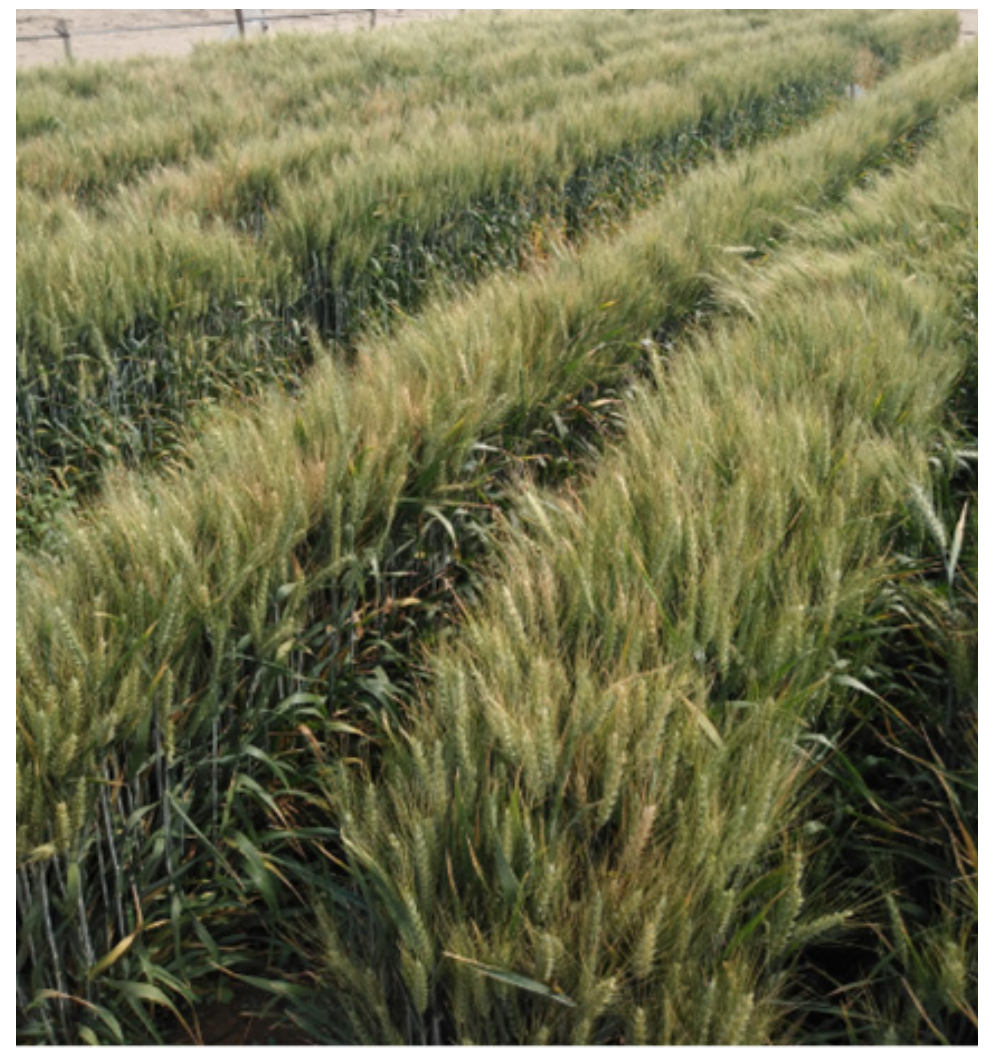

Figura 4. Maduración.

RESULTADOS Y DISCUSIÓN

En este estudio en todos análisis de varianza la interacción de los factores $S^{\star} L$ no resulto significativa para ninguna de las variables (Tabla 3 y 4). Por lo tanto, según comenta Calzada (5) si el experimento no resulta significativa la interacción de los factores, las conclusiones más importantes son las que se deduzcan de los efectos principales.

Tabla 3. Cuadrados medios del análisis de variancia para rendimiento, número espigas, peso una espiga solo grano y peso espigas con grano por $\mathrm{m}^{-2}$

\begin{tabular}{|c|c|c|c|c|c|}
\hline Fuente de variación & $\begin{array}{l}\text { Grados de } \\
\text { libertad }\end{array}$ & $\begin{array}{l}\text { Rendimiento } \\
\text { (kg ha-1) }\end{array}$ & $\begin{array}{c}\text { Número } \\
\text { espigas por } \\
\mathrm{m}^{-2}\end{array}$ & $\begin{array}{l}\text { Peso una } \\
\text { espiga solo } \\
\text { grano }(\mathrm{g})\end{array}$ & $\begin{array}{l}\text { Peso espigas } \\
\text { con grano por } \\
\text { m2 (g) }\end{array}$ \\
\hline Tratamiento & 8 & $6960944^{\star \star}$ & $44323^{\star \star}$ & $0.292^{\star \star}$ & $130694^{\star \star}$ \\
\hline Bloque & 3 & 690419NS & 4840NS & $0.020 \mathrm{NS}$ & 12532NS \\
\hline $\mathbf{S}$ & 2 & $4265858 \mathrm{NS}$ & $18516^{\star \star}$ & $0.360^{\star \star}$ & $111727^{\star}$ \\
\hline $\mathbf{L}$ & 2 & $18611669^{\star \star}$ & $152756^{\star \star}$ & $0.769^{\star \star}$ & $352864^{\star \star}$ \\
\hline $\mathbf{S}^{\star} \mathbf{L}$ & 4 & $2483124 N S$ & $3011 N S$ & $0.020 \mathrm{NS}$ & 29092NS \\
\hline Error & 24 & 1709746 & 2552 & 0.030 & 28795 \\
\hline Total & 35 & & & & \\
\hline Coeficiente de variación (\%) & & $11.93 \%$ & $7.82 \%$ & $9.76 \%$ & $11.05 \%$ \\
\hline
\end{tabular}

${ }^{\star}$ Significancia estadística al 0.05 de probabilidad. ${ }^{\star}$ Significancia estadística al 0.01 de probabilidad. NS Significa no significativo. 
Tabla 4. Cuadrados medios del análisis de variancia para peso de una espiga con grano, porcentaje peso del grano en relación a su espiga, peso de una espiga sin grano y altura de planta.

\begin{tabular}{lccccc}
\hline Fuente de variación & $\begin{array}{c}\text { Grados de } \\
\text { libertad }\end{array}$ & $\begin{array}{c}\text { Peso de una } \\
\text { espiga con } \\
\text { grano }(\mathbf{g})\end{array}$ & $\begin{array}{c}\text { Porcentaje peso del } \\
\text { grano en relación a su } \\
\text { espiga }\end{array}$ & $\begin{array}{c}\text { Peso de una } \\
\text { espiga sin } \\
\text { grano }(\mathbf{g})\end{array}$ & $\begin{array}{c}\text { Altura de } \\
\text { planta }(\mathbf{m})\end{array}$ \\
\hline Tratamiento & 8 & $0.388^{* \star}$ & $0.002 \mathrm{NS}$ & $0.031 \mathrm{NS}$ & $0.001 \mathrm{NS}$ \\
Bloque & 3 & $0.064 \mathrm{NS}$ & $0.001 \mathrm{NS}$ & $0.000 \mathrm{NS}$ & $0.000 \mathrm{NS}$ \\
$\mathbf{S}$ & 2 & $0.435^{\star \star}$ & $0.003 \mathrm{NS}$ & $0.045 \mathrm{NS}$ & $0.001 \mathrm{NS}$ \\
$\mathbf{L}$ & 2 & $0.989^{* *}$ & $0.001 \mathrm{NS}$ & $0.050 \mathrm{NS}$ & $0.003^{*}$ \\
$\mathbf{S}^{\star} \mathbf{L}$ & 4 & $0.063 \mathrm{NS}$ & $0.002 \mathrm{NS}$ & $0.015 \mathrm{NS}$ & $0.000 \mathrm{NS}$ \\
Error & 24 & 0.073 & 0.002 & 0.017 & 0.001 \\
Total & 35 & & & & \\
Coeficiente de variación (\%) & & $11.07 \%$ & $5.63 \%$ & $18.82 \%$ & $2.97 \%$ \\
\hline
\end{tabular}

${ }^{\star}$ Significancia estadística al 0.05 de probabilidad. ${ }^{\star \star}$ Significancia estadística al 0.01 de probabilidad. NS Significa no significativo.

\section{Rendimiento (kg ha-1)}

A pesar que en el análisis de varianza no se obtuvo un resultado significativo en " $\mathrm{S}$ " Distanciamiento entre semillas, pero si en "L" número de líneas (Tabla 3) se le realizo la prueba Duncan al 0.05 de significación en el factor distanciamiento entre semillas a 1.0 y $2.0 \mathrm{~cm}$ con 11513.89 y $11044.44 \mathrm{~kg} \mathrm{ha}^{-1}$ respectivamente (S1 y S2) se logró el mejor rendimiento esto debido al mayor distanciamiento entre plantas y tener menos competencia por agua, luz, nutrientes (Tabla 5).

Con cuatro líneas de trigo (L4) con 12343.75 $\mathrm{ha}^{-1}$ se obtuvo el mejor resultado en rendimiento, independientemente de la distancia entre semillas empleada (S).

Tabla 5. Comparación de medias para Rendimiento, número de espigas por $\mathrm{m}^{-2}$, peso de una espiga solo grano, peso de espigas por $\mathrm{m}^{-2}$.

\begin{tabular}{|c|c|c|c|c|}
\hline Factores & $\begin{array}{l}\text { Rendimiento } \\
\text { (kg ha-1) }\end{array}$ & $\begin{array}{l}\text { Numero de espigas } \\
\text { por } m^{-2}\end{array}$ & $\begin{array}{l}\text { Peso de una espiga } \\
\text { solo grano }(\mathrm{g})\end{array}$ & $\begin{array}{l}\text { Peso de espigas con } \\
\text { grano por } \mathrm{m}^{-2}(\mathrm{~g})\end{array}$ \\
\hline Factor S & \multicolumn{4}{|c|}{ Comparación de medias $\mathbf{S}$} \\
\hline S 0.5 & $10329.86 \mathrm{~b}$ & $681.42 \mathrm{a}$ & $1.58 \mathrm{c}$ & $1450.83 \mathrm{~b}$ \\
\hline S 1.0 & 11513.89 a & $653.33 \mathrm{a}$ & $1.78 \mathrm{~b}$ & $1640.56 \mathrm{a}$ \\
\hline S 2.0 & $11044.44 \mathrm{a}$ & $603.83 \mathrm{~b}$ & $1.93 \mathrm{a}$ & $1515.10 \mathrm{a}$ \\
\hline Factor L & \multicolumn{4}{|c|}{ Comparación de medias $\mathbf{L}$} \\
\hline L2 & $9925.00 \mathrm{~b}$ & $517.42 b$ & $2.00 \mathrm{a}$ & $1381.18 \mathrm{~b}$ \\
\hline L4 & $12343.75 \mathrm{a}$ & $693.5 \mathrm{a}$ & $1.79 \mathrm{~b}$ & $1720.10 \mathrm{a}$ \\
\hline L6 & $10619.44 \mathrm{~b}$ & $727.67 \mathrm{a}$ & $1.50 \mathrm{c}$ & $1505.21 \mathrm{~b}$ \\
\hline
\end{tabular}

Medias con diferente letra son significativamente diferentes (prueba de Duncan al 0.05 de probabilidad). 


\section{Numero de espigas por $\mathbf{m}^{-2}$}

La mayor cantidad de espigas $\mathrm{m}^{-2}$ fue registrada al sembrar entre semillas a 0.5 y a $1.0 \mathrm{~cm}$ con 681.42 y 653.33 número de espigas por $\mathrm{m}^{-2}$ respectivamente (S0.5 y $\mathrm{S} 1.0$ ) superando estadísticamente al S 2.0 con 603.83 número de espigas por $\mathrm{m}^{-2}$, respecto al número de líneas 4 y 6 líneas con 693.5 y 727.67 número de espigas por $\mathrm{m}^{-2}$ también registra un mayor número de espigas por $\mathrm{m}^{-2}$. superando al factor L2 con 517.42 número de espigas por $\mathrm{m}^{-2}$ en ambos casos tanto la distancia entre semillas y número de líneas al aumentar la densidad aumenta el número de espigas por $\mathrm{m}^{-2}$ (Tabla 5).

\section{Peso una espiga solo grano (g)}

Si solamente se toma en cuenta el grano por espiga tanto para el distanciamiento entre semillas de $2.0 \mathrm{~cm}$ (S2) con 1.93 gramos y con 2 líneas (L2) con 2.00 gramos solo grano por espiga ocuparon los primeros lugares, en cambio, los factores que representan la mayor densidad con distanciamiento entre semillas de $0.5 \mathrm{~cm}$ (S0.5) con 1.58 y con 6 líneas (L6) con 1.50 gramos solo grano por espiga ocuparon el último lugar debido a exceso de densidad y competencia entre plantas (Tabla 5).

\section{Peso de espigas con grano por $\mathrm{m}^{-2}(\mathrm{~g})$}

En el peso de espigas con grano por m-2 tanto para los disntaciamientos entre semillas de 1.0 y $2.0 \mathrm{~cm}$ con 1640.56 y 1515.10 gramos de espigas con grano por $\mathrm{m}-2$ respectivamente (S1.0 y S2.0) ocuparon el primer lugar eligiendo siempre el S2.0 para gastar menos semilla y economizar el cultivo. Respecto al numero de lineas (L) el L4 con 1720.1 gramos de espigas con grano por $\mathrm{m}^{-2}$ que ocupo el primer lugar.

Tabla 6. Comparación de medias para peso de una espiga con grano, porcentaje peso del grano en relación a su espiga, peso de una espiga sin grano y altura de planta.

\begin{tabular}{ccccc}
\hline Factores & $\begin{array}{c}\text { Peso de una espiga } \\
\text { Con grano } \mathbf{( g )}\end{array}$ & $\begin{array}{c}\text { Porcentaje peso del } \\
\text { grano en relación a } \\
\text { su espiga \% }\end{array}$ & $\begin{array}{c}\text { Peso una espiga sin } \\
\text { grano }(\mathbf{g})\end{array}$ & Altura de planta $(\mathbf{m})$ \\
\hline Factor S & $2.21 \mathrm{~b}$ & Comparación de medias S \\
S 0.5 & $2.54 \mathrm{a}$ & $71.00 \mathrm{a}$ & $0.63 \mathrm{~b}$ & $0.92 \mathrm{a}$ \\
S 1.0 & $2.55 \mathrm{a}$ & $70.00 \mathrm{a}$ & $0.76 \mathrm{a}$ & $0.93 \mathrm{a}$ \\
S 2.0 & $73.00 \mathrm{a}$ & $0.69 \mathrm{a}$ & $0.92 \mathrm{a}$ \\
Factor L & $2.69 \mathrm{a}$ & Comparación de medias L & $0.94 \mathrm{a}$ \\
L2 & $2.49 \mathrm{a}$ & $72.00 \mathrm{a}$ & $0.75 \mathrm{a}$ & $0.91 \mathrm{~b}$ \\
L4 & $2.12 \mathrm{~b}$ & $72.00 \mathrm{a}$ & $0.71 \mathrm{a}$ & $0.92 \mathrm{~b}$ \\
\hline
\end{tabular}

Medias con diferente letra son significativamente diferentes (prueba de Duncan al 0.05 de probabilidad). 


\section{Peso de una espiga con grano $(\mathrm{g})$}

Respecto al peso de una espiga con grano los factores los distanciamientos entre semillas a 1.0 y $2.0 \mathrm{~cm}$ con 2.54 y 2.55 gramos de la espiga mas grano respectivamente (S1 y S2) tuvieron el mayor peso por espiga con grano, el distanciamiento entre semilla de $0.5 \mathrm{~cm}$ con 2.21 gramos de la espiga mas grano (S0.5) por exceso de densidad tuvo menor peso y quedo en ultimo lugar. Utilizando 6 lineas con 2.12 gramos de la espiga mas grano (L6) quedo en ultimo lugar debido a el exeso de densidad lo cual disminuye el peso de la espiga con grano (Tabla 6).

\section{Porcentaje peso del grano en relación a su espiga}

En el análisis de varianza no hay evidencia estadística para demostrar que en las diferentes densidades aplicadas el grano ocupa más o menos espacio al llenar la espiga en los factores $S$ y $L$. Entonces se puede decir que el peso de solamente el grano por espiga en relación al peso de la espiga con grano no cambia en relación a las diferentes densidades aplicadas (Tabla 4).

\section{Peso una espiga sin grano (g)}

En el Análisis de Varianza no se obtuvo significancia en los factores $S$ y $L$, pero aun así se aplicó la prueba Duncan al 0.05 de significación (Tabla 4). El distanciamiento entre semillas $\mathrm{S}$ 1.0 y S 2.0 con 0.76 y 0.69 gramos de una espiga sin grano respectivamente y el número de líneas L2 y L4 con 0.75 y 0.71 gramos de una espiga respectivamente sin grano ocuparon el primer lugar estadísticamente. Tanto para los distanciamientos entre semilla $\mathrm{S} 0.5 \mathrm{~cm}$ con 0.63 gramos de una espiga sin grano y número de líneas L6 con 0.63 gramos de una espiga sin grano ocuparon el último lugar (Tabla 6).

\section{Altura de planta $(\mathrm{m})$}

En cuanto al factor $\mathrm{S}$ no se obtuvo significancia en el análisis de varianza (Tabla 4), pero aun así se le realizo la prueba de Duncan al 0.05 de significación.

En el distanciamiento entre semillas S1, S2, S3 con $0.92,0.93$ y 0.92 metros de altura de planta respectivamente no hubo diferencias significativas (Tabla 6).

La máxima altura del cultivo se logró sembrando dos líneas con 0.94 metros de altura de planta (L2).

\section{Discusión}

\section{Rendimiento (kg ha-1)}

Sería conveniente sembrar a una distancia de $2 \mathrm{~cm}$ entre semillas (S2) para economizar este insumo, además de favorecer al cultivo con mayor espacio para su desarrollo. Asimismo, Suaste (1) obtuvieron el máximo rendimiento de trigo con una densidad 180 y $240 \mathrm{~kg}$ de semilla por ha-1, por lo que se podría comparar con los resultados del presente experimento a una siembra con distanciamiento entre semillas de $1 \mathrm{~cm}$ y 2 cm (S1 y S2) con cuatro líneas (L4) con 307 y $153 \mathrm{~kg}$ de semilla por ha-1 respectivamente, en el cual se obtuvo un rendimiento mayor (Tabla 2).

También cabe recalcar que la semilla usada fue de buen vigor la cual es importante para el desarrollo del cultivo como lo reportan Abati 
(4) quienes usaron semillas de alto vigor que ayuda al establecimiento del cultivo.

Una fertilización adecuada que se usó en el presente experimento ayuda al establecimiento del cultivo y a mitigar el efecto negativo de las malezas tal como lo reportan Lerner (2) que fertilidad nitrogenada y densidades siembra actuaron independientemente sobre producción de materia seca, aunque el aporte de nitrógeno aumenta la materia seca.

El distanciamiento entre semilla de $2 \mathrm{~cm}$ (S2) y con 4 líneas (L4) con 333.3 semillas por $\mathrm{m}^{-2}$ (Tabla 2) con altos rendimientos también mitigo el efecto negativo de la maleza se puede comparar a lo reportado por Weiner (3) quienes con 400 semillas por $\mathrm{m}^{-2}$ reduce la biomasa de las malezas aumentando el rendimiento hasta en un 60 por ciento más.

En este experimento la siembra económica con distanciamiento entre semilla de $2 \mathrm{~cm}$ (S2) y con 4 líneas (L4) dando 333.3 semillas por $\mathrm{m}^{-2}$ (Tabla 2) entre el 31 setiembre del año 2019 y 22 de enero del 2020 (Periodo Invierno entrando a primavera) en cambio Spink (6) reportaron que la densidad económica óptima del trigo de invierno variaba con la fecha de siembra. El óptimo medio en tres años fueron 62, 93 y 139 plantas $\mathrm{m}^{-2}$ por lo que se podría reducir la densidad por $m$ acorde a la fecha de siembra.

\section{Numero de espigas por $\mathbf{m}^{-2}$}

Se puede relacionar al rendimiento por hectárea con distanciamiento entre semilla de $2 \mathrm{~cm}$ con 603.83 (S2) y con 4 líneas 693.5 número de espigas por $\mathrm{m}^{-2}$ (L4) que son los factores con más económicos y con mayores rendimientos estadísticamente además de tener un número máximo de espigar por $\mathrm{m}^{-2}$ (Tabla 5). Los resultados de este experimento en número de espigas por $\mathrm{m}^{-2}$, S1 con $653.33 \mathrm{~m}^{-2}$ y L4 con $693.50 \mathrm{~m}^{-2}$ (Tabla5) muestra resultados similares a lo reportado por Lerner (2) que usando la densidad de 600 plantas por $\mathrm{m}^{-2}$ el número de espigas fue mayor.

Además, los distanciamientos entre semillas de 0.5 y $1 \mathrm{~cm}$ con 681.42 y 653.33 número de espigas por $\mathrm{m}^{-2}$ respectivamente (S0.5 y S1.0), con 4 y 6 líneas con 693.5 y 727.67 número de espigas por $\mathrm{m}-2$ respectivamente ( $\mathrm{L} 6$ y $L 4$ ) (Tabla 5) a pesar de su diferencia de densidades obtienen resultados sin significancia quizá debido al número de macollos producidos que regula el rendimiento. Relacionado a los que reportaron Fioreze (7) que en bajas densidades es reflejado por un alto número de macollos.

También Beres (8) encontraron que sembrando 450 semillas de trigo $\mathrm{m}^{-2}$ se obtuvo el menor porcentaje de infestación de "wheat stem sawfly" (Cephus cinctus Norton), en comparación con otras tres densidades, dicha densidad fue similar al usar los factores $\$ 1.0$ y L4 con 667.7 semillas por $\mathrm{m}^{-2}$ (Tabla 2).

Cabe recalcar que la relación del número de espigas referente a la calidad del grano, mientras lo económico seria sembrar con distanciamientos entre $1.0 \mathrm{~cm}$ y $2 \mathrm{~cm}$ (S1 y S2) con 4 líneas (L4) no siembre podría obtenerse un grano de calidad como lo reporta Zecevic (9) que mostraron que una mayor tasa de siembra tiene un efecto positivo en la calidad del grano de trigo. Mayor densidad proporciona un mayor número de macollos primarios por metro 
cuadrado, que provoca la formación de granos con mayor tamaño y masa. Estos granos tienen una proporción más favorable de proteínas de almacenamiento y almidón, que requiere una mejor calidad. En cultivos con menor densidad, mayor número de macollos secundarios, que producen pequeños granos con menos peso y menor calidad.

El experimento tambien pudo haberse favorecido en tener menos maleza en general como lo reporta Olsen (10) que la biomasa de malezas casi siempre disminuyó al aumentar la densidad del cultivo.

\section{Peso una espiga solo grano (g)}

En los resultados ganadores en los Factores S 2.0 y L2 tiene 166.7 semillas m-2 (Tabla 2) resultado comparable con Abati (4) que determinaron que una densidad de 200 semillas de trigo $\mathrm{m}-2$ favorece la producción de masa seca de plantas en la etapa fenológica de prefloración y emergencia de espiga.

Teniendo en cuenta que el mayor distanciamiento aumenta el peso de la espiga con grano en relación a un buen plan de fertilización que reportan Kristensen (11) que fertilización con nitrógeno aumento la biomasa tanto del trigo y la maleza, pero por competencia la maleza termino por disminuir.

\section{Peso de espigas con grano por $\mathbf{m}^{-2}(\mathrm{~g})$}

Respecto a numero de lineas $(\mathrm{L})$ el ganador fue el L4 con 1720.1 gramos de espigas con grano por $\mathrm{m}^{-2}$ debido a que que se encuentra en una densidad equilibrada no en exeso como el L6 con 1505.21 gramos de espigas con grano por $\mathrm{m}^{-2}$ y escasa como la L2 1381.18 gramos de espigas con grano por $\mathrm{m}-2$ que quedaron en ultimo lugar (Tabla 5).

Si se compara el distanciamiento del factor S 0.51450 .83 gramos de espigas con grano por $\mathrm{m}^{-2}$ (Tabla5) que refleja una alta densidad de plantas obtuvo el menor peso por $\mathrm{m}^{-2}$, este resultado se relaciona a lo mencionado por Fioreze (7) que la competencia entre plantas causada por el aumento en la densidad de cultivos resultó en reducción de la acumulación de materia seca de la planta.

\section{Peso de una espiga con grano (g)}

Por lo observado la densidad no solamente influye en el peso del grano sino tambien en una espiga con grano, por la cumulacion de materia seca.

La competencia entre plantas de trigo sembradas en cuatro hileras (L4) con 2.49 gramos de la espiga mas grano fue adecuada y ganadora estadísticamente (Tabla 6), por esta razón el rendimiento no fue perjudicado ya sea por exceso o déficit de plantas. Además, dicha densidad podría hacer más competitivo al cultivo con relación a las malezas. Kristensen (11) determinaron que la proporción de biomasa de malezas con relación al total (trigo y malezas) fue disminuyendo conforme la densidad y uniformidad del cultivo se incrementaba.

\section{Porcentaje peso del grano en relación a su espiga}

Se aplicó la prueba de Duncan al 0.05 de significancia en la cual no hay significancia al comparar las medias para S $0.5, \mathrm{~S} 1.0$ y S 2.0 con 71,70 y 73 por ciento respectivamente y $L 2, L 4$ y $L 6$ con 72,72 y 71 por ciento respectivamente 
(Tabla 6), esto también lo reporta Cervantes (12) en el cultivo de maíz en la cual la densidad de población media y alta (75 y 90 mil plantas $\mathrm{ha}^{-1}$ ) fueron superiores estadísticamente en el rendimiento de semilla que la densidad baja (60 mil plantas ha-1), debido al mayor número de plantas, a pesar de no haber diferencias estadísticas en los caracteres de mazorca; este incremento pudiera deberse al mayor número de ellas.

\section{Peso una espiga sin grano (g)}

Al tener mayor densidad la espiga vacía no termina de desarrollarse por completo y por ende menos granos. Además, una alta densidad también influye no solamente en la formación del grano sino de un menor follaje. Ese resultado se relaciona a los mencionado por Fioreze (7) que este comportamiento de menor follaje puede estar asociado con la acumulación de materia seca por la planta en la fase de antesis y el menor desarrollo de las hojas para las densidades de cultivo más altas.

\section{Altura de planta ( $\mathrm{m}$ )}

En el número de línea L2 fue el ganador principalmente debido principalmente al mayor espacio para su crecimiento. Sin embargo, esto no fue suficiente para obtener el mayor rendimiento porque la población de plantas fue menor en comparación con densidades de cuatro y seis líneas con 0.91 y 0.92 metros de altura de planta (L4 y L6) (Tabla 6). Resultado relacionado a lo reportado por Chura (13) que, en algunos cultivos como maíz, la altura de planta sí es una característica relacionada con el rendimiento porque plantas más grandes pueden producir más mazorcas, lo cual no sucede en trigo porque produce una sola inflorescencia por planta.

\section{CONCLUSIONES}

Una de las mejores opciones para obtener rendimientos superiores de trigo conducido bajo un sistema de riego por goteo y economizando semilla es sembrando con un distanciamiento entre semillas de $2 \mathrm{~cm}$ con $11044.44 \mathrm{~kg}$ ha- 1 y con 4 líneas con 12343.75 kg ha-1 del cultivo.

El mayor número de espigas por $\mathrm{m}-2$ se obtiene con un distanciamiento entre semilla de $0.5 \mathrm{~cm}$ y $1 \mathrm{~cm}$ con 681.42 y 653.33 número de espigas por m-2. Además, aplicando 4 y 6 líneas con 693.5 y 727.67 número de espigas por $\mathrm{m}^{-2}$.

El mejor peso del grano por espiga se obtiene con el distanciamiento entre semillas entre $2.0 \mathrm{~cm}$ con 1.93 gramos peso espiga solo grano y 2 líneas con 2.00 gramos de peso espiga solo grano.

El mayor peso de espigas por $\mathrm{m}-2$ se obtuvo con los disntaciamientos entre semillas de 1.0 y $2.0 \mathrm{~cm}$ con 1640.56 y 1515.10 peso de espigas por $\mathrm{m}-2$ respectivamente, respecto al numero de lineas el mejor fue el factor de 4 lineas con 1720.1 peso de espigas por $\mathrm{m}^{-2}$.

Peso de una espiga con grano los mejores resultados se obtuvieron con los distanciamientos entre semillas a 1.0 y 2.0 $\mathrm{cm}$ con 2.54 y 2.55 gramos de una espiga solo grano, respecto al numero de lineas los mejores resultaro se obtuvieron con 2 y 4 lineas con 2.69 y 2.49 gramos de una espiga solo grano. 
En el porcentaje del peso del grano en S 0.5 , S1.0 y S 2.0 con 71,70 y 73 por ciento respectivamente y $L 2, L 4$ y $L 6$ con 72,72 y 71 por ciento respectivametne no se ecnotro diferencias significativas.

El peso de una espiga sola sin sus granos los ganadores fueron los factores S1.0 y S 2.0 con 0.76 y 0.69 gramos de una espiga sin grano y $L 2$ y L4 con 0.75 y 0.71 gramos de una espiga sin grano.

Respecto a la altura de planta solo el factor numero de lineas mostro diferencias siendo el mejor aplicando 2 lineas con 0.94 metros de altura de planta. El factor entre semillas no mostro diferencias significativas.

\section{REFERENCIAS BIBLIOGRÁFICAS}

1. Suaste MP, Solís E, Ledesma L, De la Cruz ML, Grageda OA, Báez A. Efecto de la densidad y método de siembra en el rendimiento de grano de trigo (Triticum aestivum L .) en el Bajío, México. Agrociencia. 2013; 47:159-170.

2. Lerner SE, Mac Maney M, Tapia MR. Densidad de siembra y fertilización nitrogenada en trigo l. cultivares marcos juarez inta y norkinpan 70. Fac Agron Univ Nac La Plata. 1986; 7(1):9-16.

3. Weiner J, Griepentrog $H$, Kristensen $L$. Suppression of weeds by spring wheat Triticum aestivum increases with crop density andspatial uniformity. J. Ecol. 2001; 38(4):784-790.

4. Abati J, Brzezinski CR, Zucareli C, Foloni JSS, Henning FA. Growth and yield of wheat in response to seed vigor and sowing densities. Rev Caatinga. 2018; 31(4):891-899.
5. Calzada J. Métodos estadísticos para la investigación. Perú: Jurídica; 1970.

6. Spink JH, Semere T, Spares DL, Whaley JM, Foulkes MJ, Clare RW. Effect of sowing date on the optimum plant density of winter wheat. Ann uppl Bid. 2000; 137:179-188.

7. Fioreze $\mathrm{S}$, Rodrigues J. Componentes produtivos do trigo afetados pela densidade de semeadura e aplicação de regulador vegetal. Semin Cienc Agrar. 2014; 35(1):39-54.

8. Beres BL,Cárcamo HA, Yang RC, Spaner DM. Integrating spring wheat sowing density with variety selection to manage wheat stem sawfly. Agron J. 2011; 103:1755-1764.

9. Zecevic V, Boskovic J, Knezevic D, Micanovic D. Effect of seeding rate on grain quality of winter wheat. Chil J Agric Res. 2014; 74(1):23-28.

10. Olsen J, Kristensen L, Weiner J. Influence of sowing density and spatial pattern of spring wheat (Triticum aestivum) on the suppression of different weed species. Weed Biol Manag. 2006; 6:165-173.

11. Kristensen L, Olsen J, Weiner J. Crop density, sowing pattern, and nitrogen fertilization effects on weed suppression and yield in spring wheat. Weed Sci. 2008; 56(1):97-102.

12. Cervantes F, Covarrubias J, Rangel JA, Terrón AD, Mendoza M, Preciado RE. Densidad de población y fertilización nitrogenada en la producción de semilla híbrida de maíz. Agron Mesoam. 2013; 24(1):101-110.

13. Chura J, Tejada J. Comportamiento de híbridos de maíz amarillo duro en la localidad de La Molina, Perú. IDESIA (Arica). 2014; 32(1):113-118. 